\title{
A construção da norma algorítmica: análise dos textos sobre o Feed de Notícias do Facebook
}

Willian Fernandes Araujo

\section{Resumo}

0 artigo analisa a construção do Feed de Notícias nos textos públicos do Facebook. 0 objetivo do estudo é entender e mapear as lógicas estabelecidas nesses textos em relação ao uso e funcionamento do mecanismo. Foram analisadas 40 publicações em página institucional do Facebook, destinada a apresentar e explicar as mudanças no mecanismo. A análise foi orientada por um entendimento performativo do texto inspirado pelos estudos de ciência e tecnologia e pela Teoria Ator-Rede. Como resultado da análise, foi observada a construção de uma lógica normativa sobre 0 relacionamento entre os produtores de conteúdo e o sistema de classificação do Feed de Notícias, nomeada no estudo como norma algorítmica.

\section{Palavras-Chave}

Facebook. Feed de Notícias. Algoritmos.

\section{Introdução}

0 Feed de Notícias é a principal funcionalidade do Facebook: uma lista de publicações de amigos, produtores de conteúdos e outros integrantes desse populoso ecossistema disponível na interface inicial do serviço. Uma das principais características desse mecanismo é seu sistema de classificação: a lista de publicações criada pelo Feed de Notícias é organizada de acordo com a relevância relativa e o potencial das publicações para cada usuário ou usuária do serviço, produzindo o que o Facebook costuma chamar defeed personalizado. Nas análises públicas sobre esses processos de seleção e classificação, a figura do algoritmo tende a surgir como agente fundamental, como uma fórmula que comanda e determina como essa definição do que é relevante é feita. " 0 algoritmo do feed de notícias veicula 0 que 'pensa' ser a sua opinião e a de seus amigos e certamente não checa fatos" (CELLAN-JONES, 2016, documento online).

Introduzido no Facebook em 2006, o Feed de Notícias marca o início da transformação na lógica
Willian Fernandes Araujo I willianfaraujo@gmail.com Doutor em Comunicação e Informação pela Universidade Federal do Rio Grande do Sul (UFRGS), Brasil. 
de visibilidade e exposição dos usuários na web. No início dos anos 2000, as estruturas de sites de redes sociais colocavam grande ênfase na ideia de perfil como espaço de manifestação do indivíduo. Entretanto, a publicação de qualquer conteúdo e informação em um perfil não implicava 0 seu envio automático para os outros usuários do serviço. A introdução do Feed de Notícias significou 0 surgimento de um novo padrão que amplia a exposição de conteúdos pessoais e que foi adotado por "[...] quase todos os serviços sociais da web, do Twitter ao Instagram e Pinterest"1 (HEMPEL, 2016, documento online).

0 que um mecanismo como o Feed de Notícias faz ao definir o que é relevante para cada um de seus usuários não é algo estabelecido apenas pela sua capacidade técnica em realizar essa função. Isto é, parte-se do entendimento que 0 que os mecanismos fazem e seus usos esperados são noções definidas em processos relacionais, de negociação, que tem como parte importante a percepção dos usuários sobre o que cada funcionalidade representa e para que serve (AKRICH; LATOUR, 1992).

0 que plataformas digitais são e 0 que suas funcionalidades fazem são valores construídos também nos textos que apresentam, informam, sugerem e ensinam sobre seus mecanismos.
Serviços como Facebook costumam produzir um significativo número de publicações online, as quais buscam informar e instrumentalizar o uso de suas funcionalidades. Boas práticas, manuais ou dicas de como melhorar o uso dessas funcionalidades são alguns termos usados para designar essas publicações, geralmente disponíveis em blogs ou páginas oficiais das empresas. Esses textos representam inscrições materiais que enquadram certos entendimentos, por exemplo, classificando alguns comportamentos ou usos como bizarros, inapropriados, indesejados etc.

Diante disso, o objetivo do estudo é analisar a construção do Feed de Notícias nos textos públicos do Facebook a fim de entender que lógicas são aí construídas em relação ao uso e funcionamento do mecanismo. Para isso, foram analisadas todas as publicações na seção News Feed $F^{Y} I^{2}$ do Facebook Newsroom ${ }^{3}$, totalizando 40 textos (até julho de 2017). Essa página institucional da empresa foi criada em 2013 para apresentar e explicar as principais mudanças no mecanismo (FACEBOOK NEWSR00M, 2013). A análise foi orientada por um entendimento performativo do texto inspirado pelos estudos de ciência e tecnologia e pela Teoria Ator-Rede.

Nessa perspectiva, textos são considerados dispositivos mobilizados para estabelecer,

Todas as citações diretas de textos em língua estrangeira foram traduzidas livremente pelo autor.

Disponível em: <https://newsroom.fb.com/news/category/news-feed-fyi/>.

Disponível em: <https://newsroom.fb.com/>. 
performar ou reforçar determinada realidade, como um cenário no qual se desenvolve uma ação. Ou seja, são como mediadores que informam, moldam, estabilizam e produzem práticas sociais, servindo como agentes móveis que estendem a agência da organização. Nesse sentido, analisar esses textos representa 0 processo de descrever os roteiros inscritos neles, buscando tornar visíveis a geografia de responsabilidades e as causalidades ou conhecimento sobre 0 mundo performado nesse cenário (AKRICH, 1992).

Como resultado da análise, é observada a predominância nesses textos de uma lógica específica chamada, neste estudo, de norma algorítmica. Trata-se da definição de um regime de conhecimentos que busca normatizar 0 relacionamento entre os produtores de conteúdo e 0 sistema de classificação do Feed de Notícias do Facebook. Essa norma algorítmica costuma performar julgamentos bastante específicos sobre que tipo de conteúdo deve ser considerado normal e legítimo dentro da classificação exercida pelo feed. Em um caráter punitivo, esses enunciados costumam condicionar a visibilidade no Feed de Notícias a seguir as regras que estabelecem, ameaçando com a invisibilidade comportamentos considerados inadequados. Por fim, considera- se que a atuação do Feed de Notícias como mecanismo usado para selecionar o que será visível aos usuários do Facebook não pode ser completamente entendida sem levar em conta a mobilização desses textos que informam suas práticas, estabelecendo determinadas lógicas sobre o é considerado anormal.

\section{A lógica dos algoritmos: plataformas digitais e seus processos computacionais de classificação}

0 termo algoritmo tem apresentado sentidos interessantes para pensar as relações contemporâneas que mantemos com as tecnologias digitais. Tornou-se algo rotineiro ouvir relatos pessoais ou ler manchetes de veículos de comunicação sobre algoritmos. Por exemplo, o chamado algoritmo do Facebook é uma entidade seguidamente invocada como responsável pelas ações nessa plataforma. Em uma busca rápida pelo termo no Twitter, é possível encontrar dezenas de milhares de publicações, como a reproduzida na Figura 1, nas quais são expressas percepções de usuários comuns sobre as relações que estabelecem com a plataforma. Ou seja, como os usuários percebem o que o mecanismo faz ao definir o que será visível em seus feeds ou ao recomendar novos conteúdos, amizades, anúncios etc. 
Figura 1: Publicação de usuário ou usuária do Twitter sobre 0 'algoritmo do Facebook'4

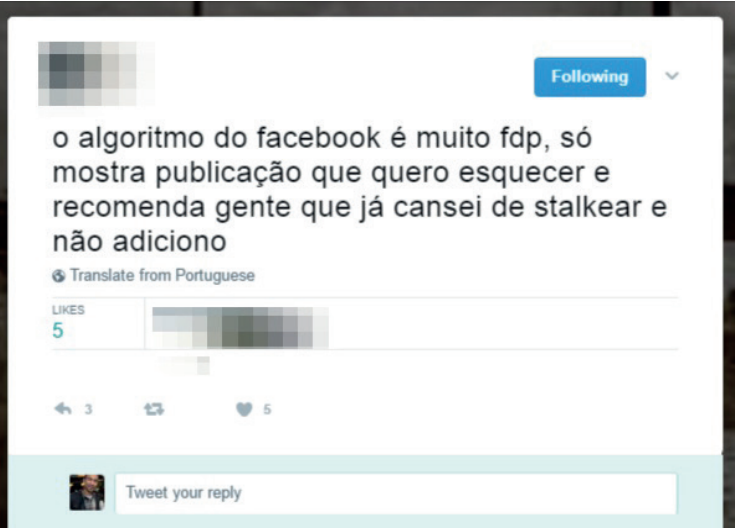

Fonte: Twitter (2017)

De percepções pessoais sobre os usos das plataformas digitais até a cobertura jornalística sobre eleições presidenciais, algoritmos têm ocupado a posição de sujeito nesses enunciados (ZIEWITZ, 2015), demonstrando a presença de processos computacionais nas práticas cotidianas. A popularização do termo algoritmo assim como 0 crescente debate em torno dos processos computacionais de classificação e organização da informação não podem ser explicados apenas pelo maior uso de tecnologias digitais. Isso se deve também a transformações na própria estrutura da web nas duas últimas décadas, que colocaram sistemas algorítmicos de classificação "[...] no centro da experiência online cotidiana dos usuários" (SANDVIG, 2014, documento online). Isto é, boa parte da experiência online atualmente (como o Feed de Notícias) é mediada por processos computacionais semiautônomos que, ao analisarem uma ampla massa de dados sobre seus usuários, estruturam como a informação é produzida, acessada, organizada, vista como legítima ou descartada como irrelevante (ANANNY, 2016). Trata-se de um padrão tecnológico contemporâneo que se dissemina pelos mecanismos que usamos diariamente para realizar diferentes práticas. Nesse sentido, o termo algoritmo tem se popularizado como uma forma de fazer referência ao poder dos processos computacionais na vida cotidiana. 0 uso do termo tem sido tão abrangente que, como sugere Ziewitz (2015, p. 12), "[...] tem tomado o lugar de conceitos como 'tecnologia', 'sistema' ou 'mídia digital'."

Na computação, algoritmo é uma entidade fundamental, que está na base de praticamente todas suas práticas (GOFFEY, 2008). Se entendermos algoritmos como um conjunto de instruções que fazem um computador realizar determinada tarefa, é possível afirmar que "Sem o algoritmo, não poderia haver computação" (GOFFEY, 2008, p. 16). Portanto, esse conceito formal de algoritmo é uma ideia abstrata por trás de praticamente todos os programas de computador que conhecemos (SKIENA, 2008). Ao mesmo tempo, nos mecanismos ditos algorítmicos com que interagimos diariamente, algoritmos são apenas parte desses processos, mas nunca o todo (DOURISH, 2016).

A forma como atuam as plataformas digitais com que interagimos não depende apenas de tarefas computacionais bem definidas e expressas em linguagem de programação. A idealização de algoritmo como uma essência formal do que fazem esses serviços, como uma fórmula 
secreta, não passa de uma simplificação que inviabiliza a análise crítica do padrão agencial desses mecanismos. Plataformas digitais como o Facebook são uma complexa confluência de aspectos diversos, computacionais ou não, como mercados financeiros, anunciantes, leis, computadores, redes, bancos de dados, servidores, etc. (BOGOST, 2015). Por isso, seria uma tarefa bastante complexa, mesmo para desenvolvedores e engenheiros, localizar o algoritmo do Facebook em algum lugar em específico nas estruturas físicas e digitais que conformam 0 Feed de Notícias.

Para Bogost (2015), a idealização de algoritmo como um objeto único, simples e fácil de delimitar escorre entre os dedos quando olhamos para a realidade empírica. A figura do algoritmo do Google, exemplifica Bogost (2015), como o agente decisivo em cada busca no serviço, desaparece quando passamos a observar a miríade de relações sociotécnicas que conformam o serviço. Não se trata de negar os importantes efeitos da agência computacional em diversos aspectos da vida coletiva, mas, sim, entendê-los como resultado de uma rede composta por pessoas, materiais, processos, máquinas, padrões, protocolos etc. As decisões incorporadas nesses mecanismos são matéria de debate, disputa e contestação e estão sempre sujeitas a testes e falhas (CRAWFORD, 2016). Por isso, Crawford (2016, p. 89) afirma que um entendimento livre de essencialismos sobre algoritmos deve expandir o horizonte de observação, incluindo "as muitas formas nas quais algoritmos são raramente estáveis e estão sempre em relação com pessoas. Ou seja, em fluxo e incorporados em espaços híbridos".

Diante desse cenário, diversos autores e autoras têm questionado o uso autoevidente da noção de algoritmo em análises sociais e críticas (BOGOST, 2015; CRAWFORD, 2016; DOURISH, 2016; GILLESPIE, 2016; KITCHIN, 2016; ZIEWITZ, 2015). Assim, optar por não tomar algoritmo como algo dado é uma postura que busca reconhecer a diversidade de sentidos que o termo pode ter em sua existência empírica. Algoritmo não é um conceito explicativo, mas, como sugere Ziewitz (2015), pode ser um conceito sensibilizador: uma noção que deriva da observação empírica de uma determinada realidade e possibilita ao pesquisador novas vias de investigação sobre seu uso (GIVEN, 2008). Tomar algoritmo como conceito sensibilizador significa mapear sua existência em um determinado contexto empírico, seguindo e registrando os valores, as lógicas e os entendimentos específicos que ele performa.

Portanto, na análise proposta neste artigo, algoritmo é observado como um conceito sensibilizador ao redor do qual é construída uma reunião material e discursiva que constitui 0 processo de classificação realizado pelo Feed de Notícias do Facebook.

\section{A performatividade do texto na construção de plataformas digitais}

0 que um mecanismo como o Feed de Notícias faz, assim como os seus usos e apropriações, 
não é algo definido apenas pela capacidade técnica do mecanismo em realizar determinada função. Isto é, o que mecanismos fazem e seus usos esperados são noções definidas em processos relacionais, de negociação, que têm como parte importante a percepção dos usuários sobre 0 que cada funcionalidade representa $\mathrm{e}$ para que serve (AKRICH, 1992). Como sugere van Dijck (2013), não é possível compreender 0 significado de compartilhar em sites de redes sociais sem acompanhar o constante processo de transformação da ideia de privacidade e do valor de estar em visibilidade. Compartilhar "não é simplesmente algo que 'está aí' na sociedade e é refletido online. Em vez disso, os proprietários e os usuários têm negociado o significado de compartilhar desde o início [do Facebook], em Harvard, em 2004, até a sua estreia na Nasdaq em 2012" (VAN DIJCK, 2013, p. 46).

Plataformas digitais são, portanto, compostas não apenas de seus arranjos materiais, mas também, de forma inseparável, de discursos que dão sustentação a determinadas práticas. Para citar um exemplo, para compreender o fenômeno da produção ubíqua de dados sobre as nossas vidas, é necessário ter em conta o imperativo da visibilidade visível nos arranjos materiais e discursivos das plataformas digitais: "somos encorajados a estar em visibilidade, a narrar e a ser calculado" (BEER, 2016, p. 148). Nesse sentido, as grandes bases de dados mantidas e alimentadas por plataformas digitais dependem desse processo pró-ativo de indução à produção de informações, corroborada por uma transformação da noção de privacidade, reforçando estar em visibilidade como um valor positivo.

Portanto, o que plataformas digitais são e 0 que suas funcionalidades fazem são valores construídos também nos textos que apresentam, informam, sugerem, ensinam sobre seus mecanismos. Serviços como Google e Facebook costumam produzir um significativo número de publicações online a fim de informar e instrumentalizar o uso de seus serviços. Por exemplo, o YouTube tem uma página de web chamada Escola de Criadores de Conteúdo ${ }^{5}$, na qual são apresentadas diferentes técnicas, práticas, dicas, sugestões de como devem ser produzidos os vídeos para a plataforma. Em um dos cursos disponíveis, é destacada a postura que deve ser assumida pelos criadores de conteúdo diante do algoritmo do YouTube: "Em vez de se preocupar com 0 que 0 algoritmo 'gosta', é melhor focar no que o público gosta. Se você fizer isso e as pessoas assistirem, 0 algoritmo seguirá essas informações" (YOUTUBE, 2017, documento online).

Boas práticas, manuais ou dicas de como melhorar 0 uso dessas funcionalidades são alguns termos usados para designar essas publicações, geralmente disponíveis em blogs ou páginas 
oficiais das empresas. Esses textos representam inscrições materiais que enquadram certos entendimentos, por exemplo, classificando alguns comportamentos ou usos como bizarros, inapropriados, indesejados etc. A partir desse entendimento, o que o Feed de Notícias faz é também definido nos textos sobre o mecanismo.

Para entender e ser capaz de analisar os enquadramentos produzidos nesses textos, é necessário tomar seu caráter performativo e, assim, abrir mão de qualquer projeto hermeneuta. Trata-se de estudar esses textos não como janelas parciais para determinada realidade, mas como mobilizações do mundo nas quais "0s nãohumanos são progressivamente inseridos no discurso" (LATOUR, 2001, p. 118). É necessário girar o foco analítico e, em vez de considerálos um reflexo distorcido da realidade, passar a seguir as formas pelas quais textos performam a realidade, por exemplo, realizando o trabalho de definição da relação entre usuários e seus feeds. Ou seja, usando a metáfora apresentada por Haraway (1992), é mudar o foco das questões de reflexo para as questões de difração $0^{6}$ é deixar de questionar se tal enunciado reproduz a realidade para mapear qual realidade produz.

Nessa perspectiva, textos são mediadores que estabelecem, performam e prescrevem determinada realidade, como um cenário no qual se desenvolve uma ação. Ao optar por essa postura, pode-se considerar que os textos não estão deslocados das práticas (NIMMO, 2011). Ao contrário, são instrumentos que informam, moldam, estabilizam e produzem práticas e, por isso, é difícil atualmente encontrar práticas dissociadas de textos. Eles "tanto registram conversações passadas como instruem novas interações situadas" (PRIMO, 2015, p. 523), servindo como agentes móveis que estendem a agência da organização.

Portanto, "textos, como inscrições materiais e móveis, são agentes ativos que reúnem, moldam e conectam práticas e, fazendo isso, também performam objetos, constituem sujeitos e inscrevem relações, domínios e fronteiras ontológicas" (NIMMO, 2011, p. 114). Assim, estudar as transformações no processo de classificação do Feed de Notícias a partir das publicações do Facebook significa seguir nessas materialidades o processo de prescrição de uma realidade específica, na qual entendimentos restritos são produzidos, como sobre a incorporação de processos algorítmicos para seleção de conteúdos considerados relevantes, mais importantes ou de qualidade.

Ao passo que esse giro do foco analítico é realizado, deixam-se de lado as questões 
hermenêuticas em favor de seguir e traçar "o trabalho de inscrição, tradução e mediação performado por textos" (NIMMO, 2011, p. 114). Trata-se de descrever os roteiros inscritos nesses textos tornando visíveis a geografia de responsabilidades que atribuem a outros (humanos ou não humanos) e as novas causalidades ou novas formas de conhecimento sobre o mundo que decorrem desse cenário (AKRICH, 1992). Logo, a proposta metodológica de estudar os textos que compõem publicamente o sistema de classificação do Feed de Notícias significa descrever como os atores são definidos, quais são seus objetivos, o que acontece após suas ações e 0 que se torna visível. Optar por seguir esses textos como mediadores vai possibilitar observar "histórias que definem quem são os atores principais, o que acontece com eles, quais são os desafios encontrados" (LATOUR, 1993, p. 9).

Nesse sentido, textos são observados como dispositivos, simultaneamente materiais e discursivos, que ajudam a transformar, reconfigurar ou produzir redes que compõem mecanismos, constituem usuários e reforçam ou reconfiguram práticas. Pensar o texto como dispositivo é reconhecê-lo não apenas como o fruto ou a representação de determinada realidade, mas também como agente produtor dessa realidade. $0 \mathrm{u}$ seja, é entender como resultado, mas também como ponto de partida (CALLON, 2002), como 0 ator que está associado a uma rede com outros atores, mas que a expande ou a atualiza. Nesse sentido, o termo dispositivos textuais é aqui usado para evidenciar o caráter inter-relacional dessas fontes empíricas. Melhor dizendo, o uso desse termo busca dar ênfase ao seu papel como mediador, que sempre é o resultado de determinado processo, mas também estabelece agenciamentos que se estendem com caráter produtivo. Logo, dispositivos textuais são redes nas quais são definidas "habilidades, ações e relações de entidades heterogêneas" (CALLON, 1990, p. 136).

A noção de dispositivos textuais é empregada para designar os textos colocados em circulação na web como agentes materiais e discursivos que atuam na conformação do que é o Feed de Notícias, performando visões particulares sobre, por exemplo, quais são as posturas consideradas aceitas diante do processo de classificação exercido pelo mecanismo. Ou seja, a análise aqui realizada reconhece e busca descrever o caráter performativo desses dispositivos na produção material e discursiva do que um mecanismo como o Feed de Notícias é e do que ele faz.

Então, dispositivos textuais é o termo usado para fazer referência à gama de conteúdos digitais nos quais o Feed de Notícias e seus componentes são paulatinamente performados, como atores que têm determinada agência, característica e relação com outros agentes incluídos nesses dispositivos.

\section{News Feed FYI e a construção da pesquisa}

Para realização da análise proposta no estudo, foram captadas, durante julho de 2017, todas

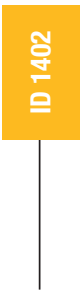


as publicações da seção News Feed $F Y T^{\top}$ do

Facebook Newsroom ${ }^{8}$, página institucional para

publicações da empresa. Criada em 2013, essa

seção é descrita como uma forma de "destacar as maiores mudanças no Feed de Notícias e explicar o pensamento por trás delas" (FACEBOOK NEWSR00M, 2013, documento online). Com 40 textos (até julho de 2017) publicados ao longo de cinco anos (Ver gráfico 1), o News Feed FYI representa um rico arquivo sobre as transformações no Feed de Notícias, assim como sobre a produção do seu sistema de classificação.

Gráfico 1: Número de publicações por ano na seção News Feed FYI do Facebook Newsroom

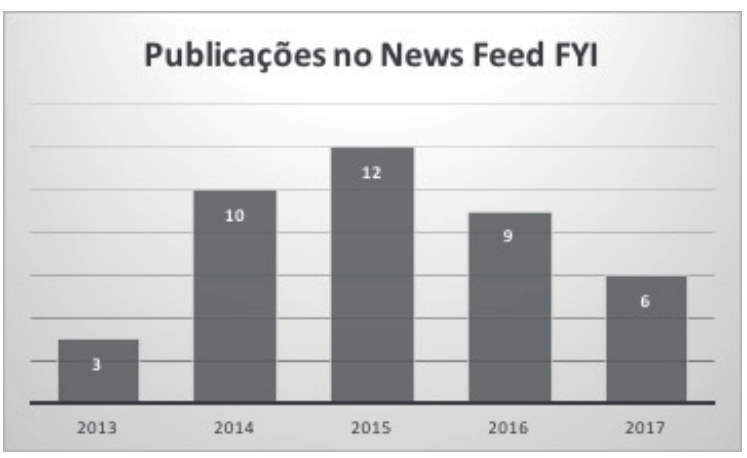

Fonte: Elaborado pelo autor

As publicações encontradas nessa página foram captadas e armazenadas usando-se o software de gestão de conteúdo digital Evernote (Figura 2). A partir do uso dessa ferramenta, foi possível organizar as publicações em ordem cronológica em um mesmo espaço digital. Ao mesmo tempo, foi possível realizar cruzamentos entre as publicações usando-se as ferramentas de pesquisa do referido software. Por exemplo, ao longo da análise, foi possível observar 0 frequente uso dos verbos afetar (to affect) e impactar (to impact) em sentenças que abordam os efeitos das transformações no Feed de Notícias. Como a Figura 2 pode ilustrar, com a ferramenta de busca desse software, foi possível constatar que, em 27 das 40 publicações, existe a utilização de sentenças que dão conta dos efeitos das transformações no mecanismo. Portanto, mais que apenas garantir 0 armazenamento e 0 acesso a conteúdo empírico, a utilização desse mecanismo proporcionou subsídios para análise produzida no estudo.

Após a captação das publicações, cada uma delas foi lida e analisada conforme 0 entendimento performativo do texto. 0 procedimento de análise realizado no estudo é inspirado pelo estudo do texto científico de Latour (1993), assim como na semiótica material de inspiração na Teoria Ator-Rede (AKRICH, 1992; AKRICH; LATOUR, 1992). Tal procedimento consiste basicamente no mapeamento da interdefinição dos atores e cadeias de tradução mobilizados nesses roteiros. Mapear a interdefinição dos atores representa traçar como os agentes são definidos no interior dessas narrativas. 
Figura 2: Reprodução da tela do software no qual foram armazenadas as publicações analisadas

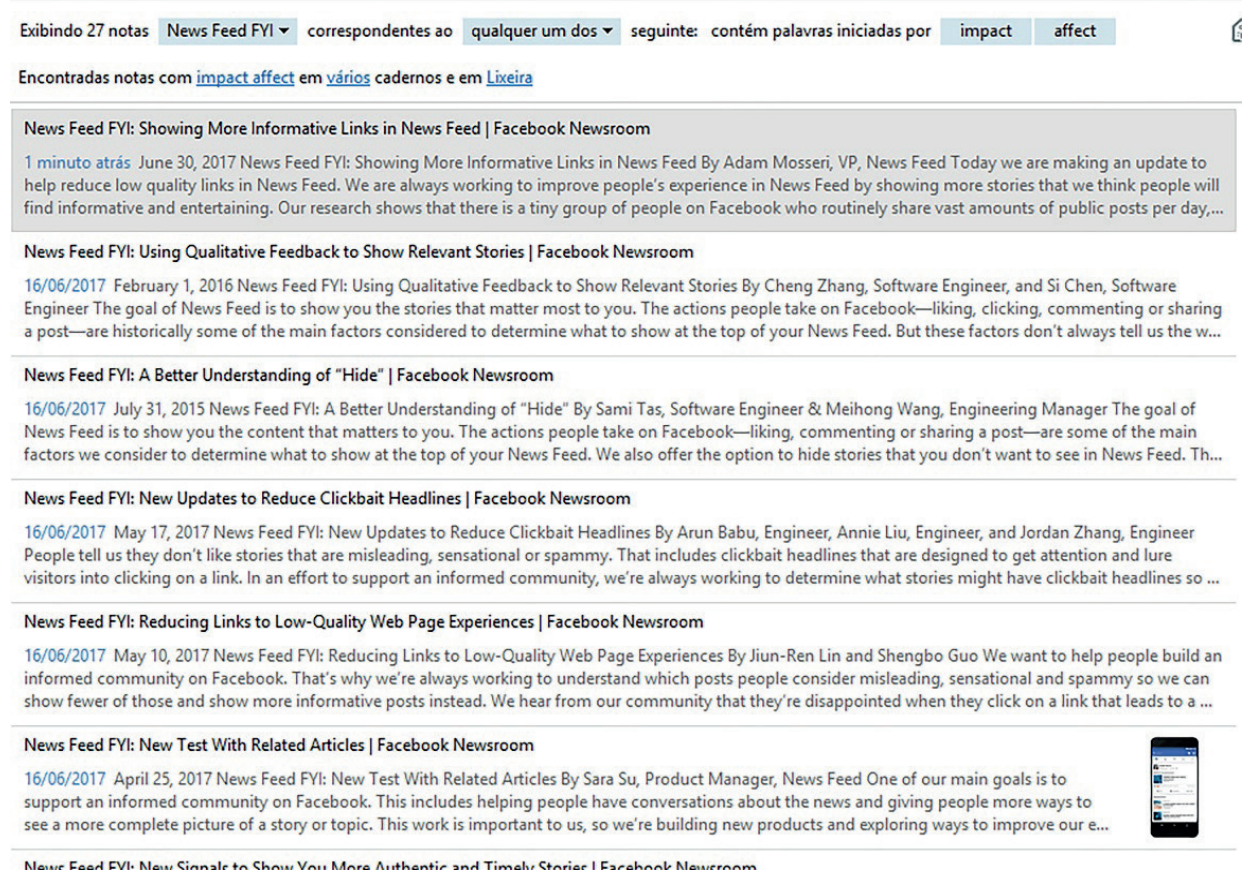

Fonte: Elaborado pelo autor

Mais que apenas enumerar os atores dessas narrativas, o objetivo é mapear como eles agem nesses roteiros e, a partir dessas ações, que relacionamento estabelecem entre si: obediência, discordância, indiferença, etc.

Cadeias de tradução é o termo usado por Bruno Latour (1993) para descrever os processos de produção de equivalência, geralmente através de dispositivos de inscrição (SCHMIDGEN, 2014). Isto é, no contexto científico, pode-se exemplificar pela tradução de determinado fenômeno em um dado, através de mecanismos de inscrição como 0 microscópio, por exemplo. Essa definição remete ao trabalho de produção de equivalências, de transformações ou deslocamentos. Trata-se do processo de composição de redes de mediação que, por exemplo, na construção do Feed de Notícias são mobilizadas para tornar conteúdo de alta qualidade equivalente aos dados de aumento do engajamento de usuários.

\section{A definição do relacionamento entre os atores}

Após a análise dos 40 textos publicados na sessão News Feed FYI do blog Facebook Newsroom, é possível destacar a proeminência de prescrições bem específicas quanto ao relacionamento do sistema de classificação do Feed de Notícias com os outros atores dessa economia da visibilidade. Nos cenários construídos nos dispositivos textuais 
analisados, as Páginas no Facebook são definidas como atores que devem produzir conteúdo de alta qualidade para permanecer ou aumentar sua visibilidade. Logo, a cada atualização ou mudança realizada nesse processo, o sentido do que é conteúdo de qualidade ou relevante é definido e reforçado por diferentes cadeias de tradução. Dessas definições é possível extrair uma lógica bastante clara quanto ao funcionamento do Feed de Notícias: atores que não produzem conteúdos de acordo com a definição de relevância ou qualidade desse sistema são afetados ou impactados com uma menor distribuição de seus conteúdos.
Ao mesmo tempo, a noção de algoritmo surge como ator decisivo do sistema de distribuição do Feed de Notícias no início do período analisado (Ver Figura 3). 0 termo é usado como um agente definidor de um conjunto de comportamentos, práticas e conteúdos que são definidos como dignos de visibilidade. "Nossa última atualização do algoritmo do Facebook ajuda a assegurar que 0 conteúdo orgânico que pessoas vejam das Páginas são os mais relevantes para eles" (KACHOLIA, 2013, documento online). Como fica claro na Figura 3, o uso do termo algoritmo se dá essencialmente nos primeiros anos do período

Figura 3: Coletânea de sentenças nas quais o termo algoritmo é empregado nos textos analisados

Ano: 2015

Título da publicação: News Feed FYI: Using Surveys to Better Understand Viral Stories | Facebook Newsroom

"As we work to improve News Feed, we make updates to the algorithm that help make sure you are seeing the most relevant stories at the top of your feed".

Ano: 2015

Título da publicação: News Feed FYI: Exposure to Diverse Information on Facebook | Facebook Newsroom

"People increasingly turn to social networks for news and information. This has led to questions about whether this creates filter bubbles where people only see information from like-minded individuals, and whether algorithms used to rank search results and social media posts contribute to this."

Ano: 2014

Título da publicação: News Feed FYI: What Happens When You See More Updates from Friends I Facebook Newsroom "As a result, the latest update to News Feed ranking treats text status updates from Pages as a different category to text status updates from friends. We are learning that posts from Pages behave differently to posts from friends and we are working to improve our ranking algorithms so that we do a better job of differentiating between the two types. This will help us show people more content they want to see."

Ano: 2013

Título da publicação: News Feed FYI: More Relevant Ads in News Feed | Facebook Newsroom

"We are currently working on some updates to the ads algorithm to improve the relevance and quality of the ads people see."

Ano: 2013

Título da publicação: News Feed FYI: Showing More High Quality Content | Facebook Newsroom

"Our latest update to the News Feed ranking algorithm helps ensure that the organic content people see from Pages they are connected to is the most interesting to them."

"To do this we decided to develop a new algorithm to factor into News Feed."

"Coming up with an algorithm to detect this is complex, and we will continue to refine it as we get more feedback. Once we developed this algorithm, we added it to our News Feed ranking algorithms as another factor to calculate the score of a News Feed story. We tested the new version of the ranking algorithm with a small segment of our users:" 
analisado (de 2013 a 2015), não sendo verificado posteriormente.

Há, no material empírico analisado, um conjunto de sentenças que visam a normatizar 0 relacionamento entre esses dois atores (Páginas e sistema de classificação do Feed de Notícias) por meio da definição de uma série comportamentos, ações e conteúdos definidos como aceitáveis. Essa lógica emergente dos dispositivos mobilizados tem um caráter punitivo, penalizando a subversão dessas normas com menor distribuição e, consequentemente, invisibilidade.

As evidências empíricas da definição e atualização dessas normas, como conjunto de práticas a serem seguidas, são abundantes nos dispositivos textuais analisados. Como ilustrado na Figura 2, enunciados desse tipo, empregando principalmente os verbos afetar (to affect) e impactar (to impact), estão presentes em, ao menos, 27 dos 40 dispositivos analisados. Por exemplo, em maio de 2017, uma nova atualização foi informada com o objetivo de reduzir a circulação de links para páginas de baixa qualidade. No dispositivo textual encarregado de divulgar a atualização do sistema de classificação, essa lógica punitiva fica clara na seguinte sentença: "Produtores de conteúdo que não usam links para páginas de baixa qualidade podem ver um pequeno aumento no tráfego, enquanto os que usam esses links devem ver um declínio no tráfego" (LIN; GUO, 2017, documento online).
Assim como a noção de páginas de baixa

qualidade, outros conceitos valorativos sobre os conteúdos que circulam no Feed de Notícias são definidos nos dispositivos textuais analisados. Um exemplo relevante que repetidamente aparece no material empírico analisado é a noção de caça-clique (click-baiting), como algo que busca ludibriar os usuários, fazendo-os acessar determinado conteúdo. Em 2014, uma atualização no Feed de Notícias foi anunciada no blog analisado justamente com objetivo de evitar o que foi chamado de títulos caça-clique (click-baiting headlines) em publicações de Páginas:

"Caça-clique" é quando o gestor de uma Página publica um link com um título que incentiva as pessoas a clicar para ver mais, sem dizer muita informação sobre o que vai ver. Mensagens como essas tendem a ter muitos cliques, o que significa que serão apresentadas a mais pessoas, ficando no topo do Feed de Notícias. (EL-ARINI; TANG, 2014, documento online).

Logo, ao definir que postagens com títulos caça-clique são entidades infringentes ao código performado pelo sistema de classificação do Facebook, essas publicações passam a ser punidas com a invisibilidade. $\mathrm{Ou}$, nos termos do dispositivo, essas Páginas "devem ver sua distribuição cair nos próximos meses" (EL-ARINI; TANG, 2014, documento online).

Não apenas tipos de conteúdo considerados inadequados são mobilizados nesses textos. Comportamentos definidos como indesejados também são definições encontradas na análise. 
Por exemplo, em junho de 2017, uma nova atualização do sistema de classificação do Feed de Notícias foi implementada com 0 objetivo de reduzir a visibilidade de usuários que "rotineiramente compartilham grandes quantidades de publicações por dia" (MOSSERI, 2017, documento online). Na publicação que divulga essa atualização, é mencionado que uma pesquisa conduzida pela empresa "mostra que links que esses usuários compartilham costumam incluir conteúdos de baixa qualidade como caça-cliques, sensacionalismo e informação equivocada" (MOSSERI, 2017, documento online). Com isso, Páginas no Facebook que têm seus conteúdos compartilhados por usuários que apresentam esse comportamento definido como inadequado "podem ver uma redução da distribuição desses links" (MOSSERI, 2017, documento online).

Outra característica relevante observada é que esses conceitos valorativos (por exemplo, caça-clique) são construídos através de cadeias de tradução que mobilizam práticas de categorização e fatores estatísticos para construi-los computacionalmente. Portanto, a definição do que é um conteúdo de baixa qualidade ou um título caça-clique é articulada em dados, pesquisas e testes, que buscam dar sustentação a enunciados como este: " $80 \%$ das vezes, pessoas preferem títulos que ajudem a decidir se querem ler todo o texto antes de clicar na postagem" (EL-ARINI; TANG, 2014, documento online).
Por fim, é possível destacar que a definição do relacionamento entre 0 sistema de classificação e as Páginas se manifesta de forma mais clara por meio de dicas ou estratégias para melhorar a distribuição, sendo essa uma noção construída com base na visibilidade (quantos usuários são alcançados por determinado conteúdo).

Você pode fazer isso ao seguir estas dicas na criação de suas publicações:

- Torne suas mensagens oportunas e relevantes

- Construa credibilidade e confiança com seu público

- Pergunte a si mesmo: "Será que as pessoas compartilhariam ou recomendariam esse conteúdo a seus amigos?"

- Pense: "Será que o meu público quer ver isso em seu Feed de Notícias?" (KACHOLIA, 2013, documento online).

Portanto, o relacionamento entre Feed de Notícias e produtores de conteúdos é construído nos textos analisados de forma bastante específica, agindo para reforçar conceitos valorativos performadas no mecanismo e punir usos marginais. Se o Feed de Notícias é um sistema de distribuição que prescreve uma lógica - segundo a qual estará visível quem ou o que obtiver mais reações no sistema (como cliques, curtidas e comentários) -, as definições em torno da relação com produtores de conteúdo agem para normatizar que essas reações sejam genuínas, e não fruto do apelo literal ou da geração de dúvida. Portanto, é possível identificar não só a existência de uma lógica de visibilidade, mas também de políticas de gerenciamento dessa lógica, que reforçam alguns de seus pontos, punindo condutas infringentes. 


\section{A produção de uma norma algorítmica}

Conforme a análise realizada no corpus selecionado para o estudo, há, na definição do relacionamento entre os atores, a produção de uma lógica específica, aqui nomeada como norma algorítmica: é a definição do relacionamento entre produtores de conteúdo e sistemas de classificação do Feed de Notícias no qual são definidos uma série de conteúdos, comportamentos, ações etc. consideradas como legítimas. Os enunciados integrantes dessa lógica têm duas principais características: primeiro, constroem, por meio da mobilização de diferentes atores (dados, estatísticas, comportamentos, entrevistas etc.), uma série de definições valorativas sobre conteúdos e comportamentos que devem ser banidos; segundo, condicionam a visibilidade nesse ambiente a seguir as regras estabelecidas e atualizadas na transformação do serviço.

Portanto, norma algorítmica trata-se de uma lógica punitiva que penaliza sua subversão com menor distribuição e, consequentemente, invisibilidade. Tentar enganar o sistema, solicitando explicitamente que usuários curtam uma publicação, implica a classificação como conteúdo não interessante e, portanto, menos visível. 0 termo norma é usado no sentido que é empregado na obra de Michel Foucault, que o define como a base do estabelecimento de distinções entre o que é normal, anormal, aceitável, legítimo, etc (FONSECA, 2001).
Logo, norma algorítmica representa a lógica de visibilidade estabelecida nos dispositivos empregados para construção do Feed de Notícias, normatizando, principalmente, 0 relacionamento entre produtores de conteúdo e o mecanismo.

Além da capacidade técnica dos processos computacionais empregados na seleção realizada no Feed de Notícias, a definição do que é visível nesse ambiente passa também pela construção de uma rede que visa a reforçar entendimentos específicos sobre, por exemplo, o que é relevante. Em outras palavras, o que o mecanismo faz também é produzido e reforçado por meio da mobilização de textos que informam suas práticas, estabelecendo uma lógica punitiva que condiciona a visibilidade a seguir as regras estabelecidas. Como apresentado na análise, é possível situar a chamada norma algorítmica de forma mais visível em enunciados definidos como boas práticas (FACEBOOK BUSINESS, 2015) ou na definição do impacto na visibilidade de Páginas diante das transformações no sistema de distribuição do feed (EULENSTEIN; SCISSORS, 2015). Esses enunciados, que atuam na produção de um domínio de conhecimentos específicos considerados legítimos, também definem 0 relacionamento esperado entre produtores de conteúdo e 0 Feed de Notícias.

Nos textos analisados, são performados julgamentos específicos sobre o que são conteúdos de qualidade dignos de estarem no Feed de Notícias e conteúdos indesejados, definidos, 
por exemplo, como memes ou publicações caça-cliques. De forma geral, o que é definido como normal dentro dessa lógica tende a ser designado por sua capacidade de gerar engajamento genuíno. Ou seja, aquele conteúdo que faz os usuários interagirem de forma genuína, sem explicitamente solicitar que eles curtam ou compartilhem.

No momento em que esses mecanismos passam a mensurar e classificar comportamentos, controlar e punir de acordo com o que é considerado legítimo se torna uma das práticas performadas no sistema de classificação do Feed de Notícias. 0 crescente uso de sistemas computacionais como tecnologias de governo tende a performar domínios de conhecimento que separam o que é legítimo do que deve ser considerado ilegítimo (INTRONA, 2015).

À medida que essa norma algorítmica passa a definir o que é legítimo e que, para estar em visibilidade no Feed de Notícias, é preciso seguir as regras aí estabelecidas, esse domínio de conhecimento tende a se internalizar nos sujeitos em interação com o mecanismo (INTRONA, 2015). Isso é mais visível nas práticas de profissionais de marketing e propaganda ou gestores de Páginas no Facebook. Nesse sentido, Introna (2015) afirma que 0 caráter performativo desse tipo de conhecimento pode se incorporar às formas como os sujeitos entendem suas práticas e, mesmo, a si próprios. Por isso, esses processos "podem performar novos domínios de conhecimento, os quais, se internalizados, podem produzir novas e talvez inesperadas subjetividades" (INTRONA, 2016, p. 23). De forma similar, Bucher (2012, p. 118) considera que "algoritmos, como os desenvolvidos pelo Google, moldam fundamentalmente 0 conhecimento e significado das práticas online".

Uma vez que o Facebook é considerado por muitos como principal fonte de tráfego para produtores de conteúdo (KAFKA, 2015), é possível afirmar que a racionalidade emergente da norma algorítmica passa a se incorporar nas maneiras como esses produtores agem no Facebook. Essa normalização das práticas no Feed de Notícias pode ser observada em diversos fenômenos empíricos. Para citar um exemplo, Sardá et al. (2015) destacam uma tendência de hibridização da linguagem jornalística no Facebook, chamada pelos autores de buzzfeedização, a fim de ampliar a visibilidade no Feed de Notícias e acesso aos links de conteúdos publicados.

Esse termo é usado pelos autores para designar a transformação de estratégias clássicas do jornalismo, como clareza e objetividade dos títulos, a caminho de uma aproximação de formatos característicos do BuzzFeed. Esse site norte-americano é conhecido pelo caráter viral de suas publicações (OREMUS, 2016), geralmente em formatos de listas e com títulos enigmáticos, que alcançam uma significativa distribuição em plataformas como o Facebook ao fazerem usuários interagirem com suas publicações. 
Os efeitos dessa norma algorítmica também podem ser observados com uma busca rápida na web: é possível listar inúmeras páginas de web sobre conhecimentos em torno da figura do algoritmo do Feed de Notícias. Geralmente voltados a profissionais de marketing e propaganda, esses conteúdos posicionam 0 algoritmo como agente poderoso e, por sua vez, sugerem relações diversas com esse ator: seguir suas normas, conhecê-lo, respeitá-lo, enganálo etc. Isto é, enquanto parte dos conteúdos sugere seguir as normas a fim de não prejudicar uma marca (KELLY, 2016), outra parte propõe enganar ou hackear o algoritmo (KURTZ, 2013; OLHAR DIGITAL, 2014).

Ao mesmo tempo, essa relação entre norma algorítmica e os usos do Feed de Notícias é marcada pelo dinamismo e pela transformação. Ficam claras, na análise dos textos do corpus do estudo, as frequentes transformações e atualizações promovidas no sistema de classificação do Feed de Notícias a fim de barrar trapaças.

\section{Considerações finais}

0 estudo teve como objetivo analisar as publicações que têm, ao longo dos últimos anos, construído o Feed de Notícias e suas práticas nos espaços organizacionais do Facebook. A análise foi orientada por um entendimento performativo do texto inspirado pelos estudos de ciência e tecnologia e pela Teoria Ator-Rede.
Na perspectiva construída nesse estudo, textos são considerados dispositivos mobilizados para estabelecer, performar ou reforçar determinada realidade, como um cenário no qual se desenvolve uma ação.

A análise realizada apresentou uma série de características e recorrências das narrativas construídas no corpus estudado. De forma principal, destacou-se a definição do relacionamento entre produtores de conteúdo e 0 sistema de classificação do Feed de Notícias. A construção e definição desse relacionamento foi chamada de norma algorítmica: um regime de conhecimentos que performa julgamentos bastante específicos sobre o que é moralmente considerado normal por meio de uma lógica punitiva que ameaça produtores de conteúdo com a invisibilidade.

Por isso, considera-se possível afirmar que a forma como agem sistemas técnicos como o Feed de Notícias também é produzida e reforçada na mobilização de textos que informam suas práticas, estabelecendo determinadas lógicas sobre, por exemplo, o que é considerado anormal. Logo, além da própria capacidade técnica dos processos computacionais, a definição do que é visível em ambientes como o Feed de Notícias passa também pela construção de uma rede que visa a normalizar práticas e comportamentos. 


\section{Referências}

AKRICH, M. The De-scription of Techinical Objects. In: BIJKER, W. E.; LAW, J. (Org.). Shaping Technology / Building Society: Studies in Sociotechnical Change. Cambridge: The MIT Press, 1992, p. 205-224.

; LATOUR, B. A Summary of a Convenient

Vocabulary for the Semiotics of Human and Nonhuman Assemblies. In: BIJKER, W. E.; LAW, J. (Org.).

Shaping Technology / Building Society: Studies in Sociotechnical Change. Cambridge: The MIT Press, 1992, p. 259-264.

ANANNY, M. Toward an ethics of algorithms: convening, observation, probability, and timeliness. Science, Technology \& Human Values, v. 41, n. 1, p. 93-117, 2016.

BEER, D. Metric Power. Londres: Palgrave Macmillan, 2016.

BOGOST, I. The Cathedral of Computation. The Atlantic, [S.l.], 2015. Disponível em: < http://www. theatlantic.com/technology/archive/2015/01/thecathedral-of-computation/384300/> . Acesso em: 18 nov. 2016.

CALLON, M. Technoeconomic networks and irreversibility. The Sociological Review, 1990. v. 38, n. S1, p. 132-161.

Writing and (re) writing devices as tools for managing complexity. In: LAW, J.; MOL, A. (Org.). Complexities: social studies of knowledge practices. Durham: Duke University Press, 2002, p. 191-214.

CELLAN-JONES, R. Como o Facebook pode ter ajudado Trump a ganhar a eleição. Folha de São Paulo, [S.l.], 2016. Disponível em: < http://wwwl.folha.uol.com.br/ mundo/2016/11/1831829-como-o-facebook-pode-terajudado-trump-a-ganhar-a-eleicao.shtml > . Acesso em: 18 jul. 2017.

CRAWFORD, K. Can an Algorithm be Agonistic? Ten Scenes from Life in Calculated Publics. Science,
Technology \& Human Values , v. 41, n. 1, p. 77-92, 2016.

DIJCK, J. VAN. The culture of connectivity: A critical history of social media. [S.l.]: Oxford University Press, 2013.

DOURISH, P. Algorithms and their others: Algorithmic culture in context. Big Data \& Society. v. 3, n. 2, 2016.

EL-ARINI, K.; TANG, J. News Feed FYI: Click-baiting. Facebook Newsroom, [S.l.], 2014. Disponível em: < http://newsroom.fb.com/news/2014/08/news-feed-fyiclick-baiting/> . Acesso em: 18 jan. 2017.

EULENSTEIN, M.; SCISSORS, L. News Feed FYI: Balancing Content from Friends and Pages. Facebook Newsroom, [S.l.], 2015. Disponível em: < http:// newsroom.fb.com/news/2015/04/news-feed-fyibalancing-content-from-friends-and-pages/> . Acesso em: 5 dez. 2016 .

FACEBOOK BUSINESS. Page posting tips and best practices. [S.l.], 2015. Disponível em: < https:// www.facebook.com/business/a/page-posting-tips? mref=message_bubble $>$. Acesso em: 5 dez. 2016.

FACEB00K NEWSR00M. Announcing News Feed FYI: A Series of Blogs on News Feed Ranking. Facebook Newsroom, [S.l.], 2013. Disponível em: < https:// newsroom.fb.com/news/2013/08/announcing-newsfeed-fyi-a-series-of-blogs-on-news-feed-ranking/> . Acesso em: 14 ago. 2017.

FONSECA, M. Foucault e o direito. [S.l.]: [s.n.] Universidade de São Paulo, 2001.

GILLESPIE, T. Algorithms. In: PETERS, B. (Org.). Digital Keywords: A Vocabulary of Information Society and Culture. Nova Jersey: Princeton University Press, 2016, p. 18-30.

GIVEN, L. M. The Sage encyclopedia of qualitative research methods. Thousand 0aks: Sage Publications, 2008.

GOFFEY, A. Algorithm. In: FULLER, M. (Org.). 
Software studies: a lexicon. Cambridge: The MIT Press, 2008, p. 15-20.

HARAWAY, D. The Promises of Monsters: A Regenerative Politics for inappropriate/d Others. In: GROSSBERG, L.; NELSON, C.; TREICHLER, P. (Org.). Cultural Studies. Nova York: Routledge, 1992, p. 295-337.

HEMPEL, J. Facebook's News Feed Turns 10.

Backchannel, [S.l.], 2016. Disponível em: < https:// backchannel.com/facebooks-news-feed-turns-10cddfcla4f0fb\#.389ftopxw>.

INTRONA, L. D. Algorithms, Governance, and Governmentality: On Governing Academic Writing. Science, Technology \& Human Values, 2015. p. 0162243915587360-. Disponível em: < http://sth.sagepub.com/content/ early/2015/06/02/0162243915587360.abstract $>$.

KACHOLIA, V. News Feed FYI: Showing More High Quality Content. Facebook Newsroom, [S.l.], 2013. Disponível em: < https://www.facebook.com/business/ news/News-Feed-FYI-Showing-More-High-QualityContent>. Acesso em: 18 jan. 2017.

KAFKA, P. Meet the Man Who Fills Your News Feed: Facebook Product Boss Chris Cox (Video). Recode, [S.l.], 2015. Disponível em: < http://www.recode. net/2015/2/26/11559426/meet-the-man-who-fills-yournews-feed-facebook-product-boss-chris-cox $>$. Acesso em: 6 jan. 2017.

KELLY, L. Lisa Kelly. Twitter, [S.l.], 2016.

Disponível em: < https://twitter.com/lisamichele21/ status/783622050741121024> . Acesso em: 28 dez. 2016.

KITCHIN, R. Thinking critically about and researching algorithms. Information, Communication \& Society, v. 20, n. 1, p. 14-29, 2016.

KURTZ, J. Como burlar o algoritmo do feed de notícias do Facebook usando listas. TechTudo, [S.l.], 2013. Disponível em: < http://www.techtudo.com.br/dicase-tutoriais/noticia/2013/06/como-burlar-0-algoritmo- do-feed-de-noticias-do-facebook-usando-listas.html > . Acesso em: 5 dez. 2016.

\section{LATOUR, B. The pasteurization of France.}

Cambridge: Harvard University Press, 1993. . A esperança de Pandora: ensaios sobre a realidade dos estudos científicos. Bauru: Edusc, 2001.

LIN, J.-R.; GUO, S. News Feed FYI: Reducing Links to Low-Quality Web Page Experiences. Facebook Newsroom, [S.l.], 2017. Disponível em: < https:// newsroom.fb.com/news/2017/05/reducing-links-tolow-quality-web-page-experiences/> . Acesso em: 25 jun. 2017.

MOSSERI, A. News Feed FYI: Showing More Informative Links in News Feed | Facebook Newsroom. Facebook Newsroom, [S.l.], 2017. Disponível em: < https:// newsroom.fb.com/news/2017/06/news-feed-fyi-showingmore-informative-links-in-news-feed/> . Acesso em: 28 jul. 2017.

NIMM0, R. Actor-network theory and methodology: Social research in a more-than-human world.

Methodological Innovations Online, 2011. v. 6, n. 3, p. 108-119.

OLHAR DIGITAL. Conheça ferramentas que evitam distrações no Facebook. Olhar Digital, [S.l.], 2014. Disponível em: < http://olhardigital.uol.com.br/noticia/ conheca-ferramentas-que-acabam-com-distracoes-nofacebook/42169>. Acesso em: 4 dez. 2016.

OREMUS, W. Who Controls Your Facebook Feed. Slate, [S.l.], 2016. Disponível em: < http://www. slate.com/articles/technology/cover_story/2016/01/ how_facebook_s_news_feed_algorithm_works.html $>$. Acesso em: 18 jan. 2017.

PRIM0, A. Como fala um actante coletivo? A organização Fifa encarnada por Jérôme Valcke.

Contemporânea, 2015. v. 23, p. 512-536.

SANDVIG, C. Seeing the Sort: The Aesthetic and 
Industrial Defense of "The Algorithm". Journal of the New Media Caucus, [S.I.], 2014. Disponível em: < http://median.newmediacaucus.org/artinfrastructures-information/seeing-the-sort-theaesthetic-and-industrial-defense-of-the-algorithm/> . Acesso em: 23 nov. 2016.

SARDÁ, T. et al. A buzzfeedização do jornalismo: 5 coisas que você precisa saber sobre 0 caso Zero Hora. Lumina. v. 9, n. 2, 2015.

SCHMIDGEN, H. Bruno Latour in Pieces: An

Intellectual Biography. Nova York: Fordham University Press, 2014.

SKIENA, S. S. The Algorithm Design Manual. [S.I.]: Springer London, 2008. V. 1.

TWITTER. Twitter Search, [S.l.], 2017.

Disponível em: < https://twitter.com/

search? $\mathrm{f}=$ tweets\&vertical $=$ default\& $\mathrm{q}=\% 22$ algoritmo\%20do\%20facebook $\% 22 \&$ src $=$ typd $>$. Acesso em: 17 Mar. 2017.

YOUTUBE. Pesquisa e descoberta no YouTube. Escola de Criadores de Conteúdo Pesquisar na Escola de Criadores de Conteúdo, [S.l.], 2017. Disponível em: $<$ https://creatoracademy.youtube.com/page/lesson/ discovery?ytref $=$ popular\&cid = get-discovered\&hl=ptBR\#yt-creators-strategies-1 >. Acesso em: 18 jul. 2017.

ZIEWITZ, M. Governing algorithms: myth, mess, and methods. Science, Technology \& Human Values, v. 41, n. 1, p. 3-16, 2015. 


\section{The Construction of an}

Algorithmic Norm: an Analysis of the Texts About the Facebook News Feed

\section{La construcción de la} norma algorítmica: análisis

\section{de los textos sobre el Feed} de Noticias de Facebook

\section{Abstract}

This paper analyses the construction of the News Feed in Facebook's public texts. The objective is to map the logics enacted in these texts in relation to News Feed usage and operation. Forty texts of a Facebook webpage announcing changes in the News Feed were analyzed. The study was based on a performative understanding of text inspired by science and technology studies and the ActorNetwork Theory. The analysis describes the construction of normative logic concerning the relationship between publishers and the News Feed classification system, a construction which this paper calls the algorithmic norm.

\section{Keywords}

Facebook. News Feed. Algorithms.

\section{Resumen}

El artículo analiza la construcción del Feed de Noticias en los textos públicos de Facebook. El objetivo del estudio es entender y mapear las lógicas establecidas en estos textos en relación al uso y funcionamiento del mecanismo. Se analizaron 40 publicaciones en página institucional de Facebook destinada a presentar y explicar los cambios en el mecanismo. El análisis fue orientado por un entendimiento performativo del texto inspirado por los estudios de ciencia y tecnología y por la Teoría del Actor-Red. Como resultado del análisis, se observó la construcción de una lógica normativa sobre la relación entre productores de contenido y el sistema de clasificación del Feed de Noticias, nombrado en el estudio como norma algorítmica.

\section{Palabras-clave}

Facebook. Notícias. Algoritmos. 


\section{Expediente}

A revista E-Compós é a publicação científica em formato eletrônico da Associação Nacional dos Programas de Pós-Graduação em Comunicação (Compós). Lançada em 2004, tem como principal finalidade difundir a produção acadêmica de pesquisadores da área de Comunicação, inseridos em instituições do Brasil e do exterior.

\section{E-COMPÓS I www.e-compos.org.br I E-ISSN 1808-2599}

Revista da Associação Nacional dos Programas de Pós-Graduação em Comunicação. Brasília, v.21, n.1, jan./abr. 2018. A identificação das edições, a partir de 2008 , passa a ser volume anual com três números. Indexada por Latindex I www.latindex.unam.mx

\section{CONSELHO EDITORIAL}

Ada Cristina Machado Silveira, Universidade Federal de Santa Maria, Brasil Alda Cristina Silva da Costa, Universidade Federal do Pará, Brasil Alfredo Luiz Paes de Oliveira Suppia, Universidade Estadual de Campinas, Brasil Ana Regina Barros Rego Leal, Universidade Federal do Piauí, Brasil Ana Carolina Rocha Pessôa Temer, Universidade Federal de Goiás, Brasil André Luiz Martins Lemos, Universidade Federal da Bahia, Brasil Angela Cristina Salgueiro Marques, Universidade Federal de Minas Gerais, Brasil Ângela Freire Prysthon, Universidade Federal de Pernambuco, Brasil Antonio Carlos Hohlfeldt, Pontifícia Universidade Católica do Rio Grande do Sul, Brasil Arthur Ituassu, Pontifícia Universidade Católica do Rio de Janeiro, Brasil

Bruno Campanella, Universidade Federal Fluminense, Brasil

Cláudio Novaes Pinto Coelho, Faculdade Cásper Líbero, Brasil Cárlida Emerim, Universidade Federal de Santa Catarina, Brasil Carlos Eduardo Franciscato, Universidade Federal de Sergipe, Brasil Danilo Rothberg, Universidade Estadual Paulista, Brasil Denise Tavares da Silva, Universidade Federal Fluminense, Brasil Diógenes Lycarião, Universidade Federal do Ceará, Brasil Eduardo Vicente, Universidade de São Paulo, Brasil Eliza Bachega Casadei, Escola Superior de Propaganda e Marketing - SP, Brasil Eneus Trindade, Universidade de São Paulo, Brasil

Erick Felinto de Oliveira, Universidade do Estado do Rio de Janeiro, Brasil Erly Vieira Júnior, Universidade Federal do Espírito Santo, Brasil Francisco de Assis, FIAM-FAAM Centro Universitário, Brasi

Francisco Elinaldo Teixeira, Universidade Estadual de Campinas, Brasi Francisco Gilson R. Pôrto Jr., Universidade Federal do Tocantins, Brasil Frederico de Mello Brandão Tavares, Universidade Federal de Ouro Preto, Brasil Gabriela Reinaldo, Universidade Federal do Ceará, Brasil Gilson Vieira Monteiro, Universidade Federal do Amazonas, Brasil Gustavo Daudt Fischer, Universidade do Vale do Rio dos Sinos, Brasi Itania Maria Mota Gomes, Universidade Federal da Bahia, Brasil Jiani Adriana Bonin, Universidade do Vale do Rio dos Sinos, Brasil José Afonso da Silva Junior, Universidade Federal de Pernambuco, Brasil José Luiz Aidar Prado, Pontifícia Universidade Católica de São Paulo, Brasil Josette Maria Monzani, Universidade Federal de São Carlos, Brasi Juçara Gorski Brittes, Universidade Federal de Ouro Preto, Brasil
Juliana Freire Gutmann, Universidade Federal da Bahia, Brasil Laura Loguercio Cánepa, Universidade Anhembi Morumbi, Brasil Leonel Azevedo de Aguiar, Pontifícia Universidade Católica do Rio de Janeiro, Brasil Letícia Cantarela Matheus, Universidade do Estado do Rio de Janeiro, Brasil Luciana Coutinho Souza, Universidade de Sorocaba, Brasil Maria Ataide Malcher, Universidade Federal do Pará, Brasil Maria Elisabete Antonioli, Escola Superior de Propaganda e Marketing - SP, Brasil Maria das Graças Pinto Coelho, Universidade Federal do Rio Grande do Norte, Brasil Marialva Carlos Barbosa, Universidade Federal do Rio de Janeiro, Brasil Marcel Vieira Barreto Silva, Universidade Federal da Paraíba, Brasil Marcia Tondato, Escola Superior de Propaganda e Marketing, Brasil Marli Santos, Universidade Metodista de São Paulo, Brasil

Márcio Souza Gonçalves, Universidade do Estado do Rio de Janeiro, Brasil Mauricio Mario Monteiro, Universidade Anhembi Morumbi, Brasil Mayka Castellano, Universidade Federal Fluminense, Brasil

Mozahir Salomão Bruck, Pontifícia Universidade Católica de Minas Gerais, Brasil Nisia Martins Rosario, Universidade Federal do Rio Grande do Sul, Brasil Paolo Demuru, Universidade Paulista, Brasil

Paula Melani Rocha, Universidade Estadual de Ponta Grossa, Brasil Potiguara Mendes Silveira Jr, Universidade Federal de Juiz de Fora, Brasil Priscila Ferreira Perazzo, Universidade Municipal de São Caetano do Sul, Brasil Rafael Cardoso Sampaio, Universidade Federal do Paraná, Brasil Rafael Tassi Teixeira, Universidade Tuiuti do Paraná, Brasil Regiane Lucas Garcês, Universidade Federal de Minas Gerais, Brasil Regiane Regina Ribeiro, Universidade Federal do Paraná, Brasil Renata Pitombo Cidreira, Universidade Federal do Recôncavo da Bahia, Brasil Renato Essenfelder, Escola Superior de Propaganda e Marketing, Brasil Roberto Elísio dos Santos, Universidade Municipal de São Caetano do Sul, Brasil Rodolfo Rorato Londero, Universidade Estadual de Londrina, Brasil Roseli Figaro, Universidade de São Paulo, Brasil

Simone Maria Andrade Pereira de Sá, Universidade Federal Fluminense, Brasil Sofia Cavalcanti Zanforlin, Universidade Católica de Brasília, Brasil Sônia Caldas Pessoa, Universidade Federal de Minas Gerais, Brasil Tatiana Oliveira Siciliano, Pontifícia Universidade Católica do Rio de Janeiro, Brasil Thaïs de Mendonça Jorge, Universidade de Brasília, Brasil

Valquiria Michela John, Universidade Federal do Paraná, Brasil

\section{CONSELHO CIENTÍFICO}

Cristiane Freitas Gutfreind, Pontifícia Universidade Católica do Rio Grande do Sul, Brasil | Eduardo Antônio de Jesus, Universidade Federal de Minhas Gerais, Brasil I Eduardo Morettin, Universidade de São Paulo, Brasil I Irene de Araújo Machado, Universidade de São Paulo, Brasil I Miriam de Souza Rossini, Universidade Federal do Rio Grande do Sul, Brasil

\section{COMISSÃO EDITORIAL}

Igor Pinto Sacramento, Universidade Federal do Rio de Janeiro, Brasil I Kelly Cristina de Souza Prudencio, Universidade Federal do Paraná, Brasil I Osmar Gonçalves dos Reis Filho, Universidade Federal do Ceará, Brasil I Rafael Grohmann, Faculdade Cásper Líbero, Brasil I Thaiane Moreira de Oliveira, Universidade Federal Fluminense, Brasil (editores associados)

\section{CONSULTORES AD HOC}

Afonso de Albuquerque, Universidade Federal Fluminense, Brasil I Cláudia Lago, Universidade de São Paulo, Brasil I Cesar Baio Santos, Universidade Federal do Ceará, Brasil I Eduardo Pellanda, Pontifícia Universidade Católica do Rio Grande do Sul, Brasi | Francisco Rüdiger, Pontifícia Universidade Católica do Rio Grande do Sul, Brasil | Karina Woitowicz, Universidade Estadual de Ponta Grossa, Brasil I Luis Mauro Sa Martino, Faculdade Cásper Líbero, Brasil I Norval Baitello Jr, Pontifícia Universidade Católica de São Paulo, Brasil I Pedro Guimarães, Universidade de Campinas, Brasil

\section{EQUIPE TÉCNICA}

ASSISTENTES EDITORIAIS Márcio Zanetti Negrini e Melina Santos । REVISÃO DE TEXTOS Fátima Áli | EDITORAÇÃO ELETRÔNICA Roka Estúdio
COMPÓS I www.compos.org.br

Associação Nacional dos Programas de Pós-Graduação em Comunicação

Presidente

Marco Roxo

Programa de Pós-Graduação em Comunicação - UFF marcos-roxo@uol.com.br

Vice-Presidente

Isaltina Gomes

Programa de Pós-Graduação em Comunicação - UFPE

isaltina@gmail.com

Secretária-Geral

Gisela Castro

Programa de Pós-Graduação em Comunicação

e Práticas de Consumo - ESPM

castro.gisela@gmail.com

CONTATO I revistaecompos@gmail.com 